\title{
Study of pneumococcal vaccine response in children with underlying medical conditions
}

\author{
Marta Garrido Jareño ${ }^{1}$, José Miguel Sahuquillo Arce ${ }^{1}$, Hector Rodríguez Vega ${ }^{1}$, Carmen \\ Lloret Sos ${ }^{1}$, Ana Gil Brusola ${ }^{1}$, José Luis López Hontangas ${ }^{1}$, Carmen Carreras Vázquez ${ }^{1}$, \\ Leonor Puchades Carrasco ${ }^{2}$, Antonio Pineda Lucena ${ }^{3}$, and Javier Peman García ${ }^{2}$ \\ ${ }^{1}$ La Fe University and Polytechnic Hospital \\ ${ }^{2}$ Health Research Institute La Fe \\ ${ }^{3}$ Centre for Applied Medical Research
}

May 10, 2021

\begin{abstract}
Background: Pneumococcal infection causes serious illness in children, especially those with underlying conditions. Although pneumococcal vaccination in the pediatric population is recommended, the immune response to pneumococcal vaccines in this group is usually deficient. The aim of this study was to study the response to pneumococcal vaccines in children with underlying conditions. Methods: A one-year retrospective study on children with underlying conditions attended at the Pediatric Immunology Unit of La Fe University Hospital (Valencia, Spain). Patient demographics, immunosuppressive diseases, pneumococcal vaccination coverage, serum levels of C3, C4, IgA, IgM, IgG, IgG1, IgG2, IgG3, IgG4, blood levels of leukocytes, neutrophils and CD4 and CD8 T lymphocyte were analyzed. Results: A total of 85 children were included in the study. Only $29.41 \%$ of them had the complete vaccination schedule, most under 5 years of age $(76.00 \%)$. Protective levels of pneumococcal antibodies were found only in $48 \%$ of the correctly vaccinated patients, whose serum levels of IgG4 and CD4/CD8 ratio were higher than in children with deficient response. Low vaccine response was mostly found among leukemic patients, who exhibited the lowest level of CD4 T lymphocyte. Patients' age, immunosuppressive therapy, and serum levels of C3, C4, IgG3 and lymphocytes were associated with pneumococcal vaccine response. Conclusion: Most of the patients lacked some dose of pneumococcal vaccine and had an inadequate response. Mathematical models can be useful in clinical practice to detect non responders. Emphasis should be placed on completing a vaccination schedule and, if necessary, revaccination with a booster dose of pneumococcal vaccines.
\end{abstract}

\section{Study of pneumococcal vaccine response in children with underlying medical conditions}

Marta Garrido-Jareño, MSc ${ }^{1,2,3^{*}}$, José Miguel Sahuquillo-Arce, $\mathrm{MD}^{2,4}$, Héctor Rodríguez-Vega, MD ${ }^{5}$, Carmen Lloret-Sos, $\mathrm{MD}^{2}$, Ana Gil-Brusola, $\mathrm{MD}, \mathrm{PhD}^{2,6}$, José Luis López-Hontangas $\mathrm{MSc}^{2,6}$, Carmen CarrerasVázquez, $\mathrm{MD}^{5}$, Leonor Puchades-Carrasco, $\mathrm{PhD}^{1}$ Antonio Pineda-Lucena, $\mathrm{PhD}^{7}$, Javier Pemán-García, MD, $\mathrm{PhD}^{2,6}$

${ }^{1}$ Drug Discovery Unit, Health Research Institute Hospital La Fe (Valencia, Spain)

${ }^{2}$ Microbiology Department, University and Polytechnic Hospital La Fe (Valencia, Spain)

${ }^{3}$ Valencian Institute of Pathology, Catholic University of Valencia (Valencia, Spain)

${ }^{4}$ Respiratory Infection Research Group, Health Research Institute Hospital La Fe (Valencia, Spain)

${ }^{5}$ Paediatric Department, University and Polytechnic Hospital La Fe (Valencia, Spain)

${ }^{6}$ Severe Infection Research Group, Health Research Institute Hospital La Fe (Valencia, Spain) 
${ }^{7}$ Molecular Therapeutics Program, Center for Applied Medical Research, University of Navarra (Pamplona, Spain)

\section{Correspondence*}

Marta Garrido Jareño

Microbiology Department, University and Polytechnic Hospital La Fe (Valencia, Spain)

Avenida Fernando Abril Martorell, 106 Torre A $3^{\text {a }}$ planta

46026 Valencia

Telephone number : 34692933193

E-mail : marta.garrido@ucv.es

Key words: Pneumococcal vaccine; Children with underlying conditions; Vaccination status; Immunoglobulin levels; Prediction of vaccine response.

\section{Abstract}

Background: Pneumococcal infection causes serious illness in children, especially those with underlying conditions. Although pneumococcal vaccination in the pediatric population is recommended, the immune response to pneumococcal vaccines in this group is usually deficient. The aim of this study was to study the response to pneumococcal vaccines in children with underlying conditions.

Methods: A one-year retrospective study on children with underlying conditions attended at the Pediatric Immunology Unit of La Fe University Hospital (Valencia, Spain). Patient demographics, immunosuppressive diseases, pneumococcal vaccination coverage, serum levels of C3, C4, $\operatorname{IgA}$, $\operatorname{IgM}$, $\operatorname{IgG}, \operatorname{IgG}_{1}, \operatorname{IgG}_{2}, \operatorname{IgG}_{3}, \operatorname{IgG}_{4}$, blood levels of leukocytes, neutrophils and CD4 and CD8 T lymphocyte were analyzed.

Results: A total of 85 children were included in the study. Only $29.41 \%$ of them had the complete vaccination schedule, most under 5 years of age $(76.00 \%)$. Protective levels of pneumococcal antibodies were found only in $48 \%$ of the correctly vaccinated patients, whose serum levels of IgG4 and CD4/CD8 ratio were higher than in children with deficient response. Low vaccine response was mostly found among leukemic patients, who exhibited the lowest level of CD4 T lymphocyte. Patients' age, immunosuppressive therapy, and serum levels of C3, C4, IgG3 and lymphocytes were associated with pneumococcal vaccine response.

Conclusion: Most of the patients lacked some dose of pneumococcal vaccine and had an inadequate response. Mathematical models can be useful in clinical practice to detect non responders. Emphasis should be placed on completing a vaccination schedule and, if necessary, revaccination with a booster dose of pneumococcal vaccines.

\section{INTRODUCTION}

Pneumococcal infection, especially invasive pneumococcal disease (IPD), includes potentially fatal conditions such as meningitis, bacteremia or pneumonia, and it accounts for about $11 \%$ of all deaths worldwide in children under 5 years of age ${ }^{1}$. According to WHO reports ${ }^{2}$, Streptococcus pneumoniae caused about 14.5 million cases of serious illness and 735,000 deaths in non-HIV children under 5 in the year 2000. Regardless of age, the most vulnerable groups comprise mainly patients who are immunocompromised due to splenectomy, drepanocytosis, bone marrow transplant, HIV infection, chronic inflammatory diseases or are under immunosuppressive treatment (IT) ${ }^{3,4}$.

Pneumococcal vaccines have directly decreased the rates of IPD on vaccinated children and indirectly through herd immunity- on unvaccinated children ${ }^{5}$. However, the immune response to pneumococcal vaccines in immunocompromised children (IC) is reportedly lower than in healthy children, especially when conjugated vaccines are employed ${ }^{6}$. 
Consequently, the Advisory Committee on Vaccines (ACV) of the Spanish Association of Pediatrics recommends special vaccination schedules for IC based on the routinely use of pneumococcal conjugated vaccines in combination with booster doses of polysaccharide vaccines, but also for immunocompetent children with underlying chronic medical conditions ${ }^{7}$. The main difference being the greater number of pneumococcal vaccine shots received by this particular group of patients (Table $\mathrm{S}^{7}$ ).

Despite the extensive use of pneumococcal vaccines worldwide, measuring pneumococcal antibody responses is still problematic, especially the assessment and detection of serotype-specific antibodies ${ }^{8}$. In addition, immune responses present considerable inter-individual variation, particularly in IC, and some studies relate pneumococcal vaccine failure to co-morbidities ${ }^{9,10}$.

Cytokines, major immunoglobulin classes (IgG, IgA, IgM), complement system and white blood cells play central roles in a proper protective immune response, including that to pneumococcal vaccines ${ }^{11}$. Thus, serum levels of these components could be used to identify low or non-responder patients who could benefit from an alternative vaccination schedule.

Systems biology approaches and mathematical modelling are increasingly being used to improve the understanding and the prediction of biological processes ${ }^{12,13}$. Several models to estimate the impact of the immunogenicity and safety of pneumococcal vaccination in IC patients have been published over the last few years, particularly on PCV13 ${ }^{14-16}$; but, the relationship between the serum proteins involved in the immune response and the pneumococcal vaccine should be studied in more detail to assist clinical management, particularly in IC and in children with underlying conditions. Under this rationale, we have developed a mathematical model to predict pneumococcal vaccine responses in children with previous immunological alterations to guide clinical decision-making based on the baseline immunological status of each patient and reach a personalized medicine.

\section{MATERIALS AND METHODS}

\subsection{Study design}

An observational retrospective study was performed in La Fe University Hospital from November 2018 to November 2019. Inclusion criteria were patients under 16 years of age attended at the Pediatric Immunology Unit who had had a pneumococcal serology performed during the study period.

Children were classified in 8 groups according to their main clinical diagnosis: a) Chronic pulmonary disease (CPD): bronchiectasis, bronchitis and asthma; b) Recurrent non-pulmonary infections (RI): diarrhea, meningitis and blood stream infections; c) Disorders of blood components (DBC): sickle cell anaemia, thrombocytopenic purpura and neutropenia; d) Leukemia and hematopoietic cell transplantation (LHT); e) DiGeorge syndrome (DS); f) Primary immunodeficiency (PI): hypogammaglobulinemia, chronic granulomatous disease and ataxia-telangiectasia; g) Autoimmune diseases (AD): Crohn's disease and severe atopic dermatitis; and h) Miscellanea (MIS); prolonged fever, facial cellulitis, Osler-Weber-Rendu Disease, lymphadenopathies and Turner-Syndrome.

The analysis of serum samples was performed at the Microbiology Department and the Clinical Analysis Department. The clinical information on the pneumococcal vaccination coverage of the patients was obtained from the Nominal Vaccine Registry (NVR) as registered by the Conselleria de Sanitat of the Generalitat Valenciana. Patient recruitment and sampling procedures were performed in accordance with the Declaration of Helsinki and applicable local regulatory requirements and laws after approval from the Ethics Committee of the Health Research Institute Hospital La Fe.

The database included patient demographics, clinical data associated with the diagnosis (i.e., immunosuppressed disease, IT, previous IPD, pneumococcal vaccination coverage, etc.), and analytical data (serum levels of $\mathrm{C} 3, \mathrm{C} 4$, IgA, IgM, IgG, IgG ${ }_{1}, \operatorname{IgG}_{2}, \operatorname{IgG}_{3}, \operatorname{IgG}_{4}$; and blood levels of leukocytes, neutrophils and CD4 and CD8 T lymphocytes).

\subsection{ELISA assays}


The VaccZymeTM Anti-PCP IgG Enzyme Immunoassay Kit (The Binding Site Group, Birmingham, UK) was used according to the manufacturer's instructions. Patients were classified into two groups according to their Anti-PCP IgG concentration as measured by ELISA: pneumococcal vaccine responders ( $\operatorname{IgG}>50$ $\mathrm{mg} / \mathrm{L}$ ) and non-responders (IgG [?]50 mg/L) ${ }^{17,18}$.

\subsection{Statistical Analysis}

The ANOVA Test was used to compare the means of anti-PCP IgG, C3, C4, IgA, IgM, IgG, IgG , IgG $_{2}$, $\mathrm{IgG}_{3}, \mathrm{IgG}_{4}$, leukocytes, neutrophils, CD4 and CD8 $\mathrm{T}$ lymphocytes and $\mathrm{CD} 4 / \mathrm{CD} 8$ ratios of the 8 groups. The Student's t-test was used to compare the means according to the vaccine response profile in patients with correct immunization schedule. Fisher's exact test was used to compare categorical variables.

Multiple linear regression backward elimination was performed to predict pneumococcal vaccine response. The variables used in the analysis were age, sex, C3, C4, IT, IgA, IgM, IgE, IgG, IgG1, IgG2, IgG3, IgG4, total leukocytes count, total lymphocytes count, monocytes, eosinophils, basophils, lymphocytes TCD8, lymphocytes TCD4 and CD4/CD8 ratio. $P$-values $<0.05$ were considered statistically significant.

\section{RESULTS}

\subsection{Results according to patient's age}

A total of 85 patients were included during the study period. The mean age was $7.17+-4.76$ years. More than half of the patients were boys (67.06\%). Vaccination profiles, IPD history and anti-PCP IgG levels according to patients' age, sex and principal diagnosis are summarized in Table 1.

Conjugated and polysaccharide vaccines were used alternately depending on the year of inoculation. Combinations of conjugated vaccines were common, especially with PCV13 and PCV7 in older patients. Nevertheless, PCV13 was the vaccine most frequently used (76.50\%), followed by PCV7 (28.24\%), PPSV23 (11.76\%), and PCV10 (2.35\%).

Six patients had been diagnosed with IPD within the previous two years, most in the group of 10-16 years, yet one of them had a complete immunization schedule. Patients with previous IPD did not achieve higher anti-PCP IgG levels than patients without IPD $(70.92+-32.54$ vs $73.70+-77.20$; T-test $=0.254 ; p=0.399)$.

An inverse correlation between patient's age and anti-PCP IgG titer was observed. Older patients presented lower level of pneumococcal antibodies, although no statistically significant differences were found (ANOVA test $=0.951 ; p=0.417)$.

The immunization schedule was correct only in 25 patients $(29.41 \%)$, nineteen of whom were under 5 years of age $(76.00 \%)$. Deficient production of pneumococcal antibodies in patients with correct immunization schedule was detected in 12 of these patients. Children under 2 years presented the highest vaccination coverage rate $(94.44 \%)$; however, almost half of them $(41.17 \%)$ were non-responders. Children older than 2 did not reach a vaccine coverage greater than $15 \%$.

Most of the patients inadequately vaccinated were one dose short of PSSV23 to properly complete the pneumococcal vaccination schedule $(85.00 \%)$. Additionally, patients with a complete immunization schedule did not present greater pneumococcal vaccine response rates than children without complete immunization schedule $\left(48.00 \%\right.$ vs $\left.48.33 \%, \mathrm{Chi}^{2}=0.04 ; p=0.841\right)$.

Figure 1 represents the mean levels of serum components and CD4/CD8 ratio of the twenty-five patients with complete pneumococcal vaccination according to vaccination response. In children with pneumococcal vaccine response, the level of serum components was higher in all cases except for IgG1 were it was lower, both groups being within the normal IgG1 range. Statistically significant differences were found only for IgG4 (T Test $=-2.31, p=0.049)$ and CD4/CD8 ratios $(\mathrm{T}$ Test $=-2.28, p=0.033)$.

\subsection{Results according to the main clinical diagnosis}


PI and RI were the most common disorders and patients were not adequately vaccinated, regardless of the main diagnostic group (70.58\%) However, the patients with correct vaccine response belonged mainly to the RI group (38.46\%) followed by the PI group (23.07\%).

Patients from the RI group presented the highest level of anti-PCP IgG (112.07 +- 109.07), whereas the lowest was found in the LHT group $(25.36+-17.84)$. No statistically significant differences were found when the means of anti-PCP IgG level of the 8 groups were compared (ANOVA test $=1.206 ; p=0.092$ ).

Within properly vaccinated children, the LHT group presented the lower rate of antibody response, and none of the fully vaccinated patients achieved protective levels of anti-PCP IgG.

Of the total of patients, seventeen (20\%) were receiving IT. Corticosteroids were the immunosuppressive drugs more frequently used $(70.6 \%)$, followed by methotrexate $(23.53 \%)$. TNF-alpha inhibitors were the least used $(5.88 \%)$, as summarized in Table 2 .

Significant differences were found in CD4 T lymphocyte and $\mathrm{C} 4$ levels among the 8 groups (ANOVA test $=3.349 ; p=0.004$ and ANOVA test $=12.997 ; p=0.001$, respectively; Figure 2A-B). RI patients had the highest level of CD4 T lymphocyte counts while the LHT group had the lowest. In the case of C4, the LHT group showed the highest level, while the DBC group presented the lowest. The level of the rest of the serum components and cell counts in each group is summarized in Tables S2 and S3.

\subsection{Predicting pneumococcal vaccine response}

A multivariate regression model with a $67 \%$ accuracy to predict vaccination response was created based on patients age, IT, and blood levels of $\mathrm{C} 3, \mathrm{C} 4$, IgG3 and total lymphocyte count $(\mathrm{R}=0.818$; R Square $=0.670$; Adjusted R Square=0.553; Std. Error of the estimate=0.34), as is represented in Table 3. Higher age, IT and a high $\mathrm{C} 3$ level contributed negatively to having an adequate pneumococcal vaccine response while high C4, IgG3 and lymphocyte serum levels contributed positively.

\section{DISCUSSION}

The main findings of our study are (a) pneumococcal vaccination coverage rates in our cohort were low, (b) the LHT group presented an inadequate response to vaccination, (c) low-pneumococcal antibody response in patients with correct immunization schedule appeared in more than half of the patients, (d) levels of blood components involved in immune response were higher in vaccine responders, and (e) we built a mathematical model that could predict pneumococcal vaccine response with a $67 \%$ probability.

Vaccination against pneumococcal disease is recommended in Spain according to the Advisory Committee on Vaccines of the Spanish Association of Pediatrics for all children under 5 years of age and children of any age at risk of infection due to underlying diseases. In Comunitat Valenciana, only the PCV13 is included in the childhood immunization schedule since May 2015, and consequently, it is the most found in our series ${ }^{19}$.

Older patients (10-16 years) had lower anti-PCP IgG levels and higher rates of previous IPD, possibly due to the poorer vaccination found in our cohort (29.41\%). Nevertheless, in our study, no true pneumococcal vaccine failure case was reported, despite recent series showing cases of IPD by PCV13 serotypes in children who had been age-appropriately vaccinated ${ }^{20}$.

Vaccination response was inadequate or insufficient in more than half of our patients regardless of the underlying disease, LHT being the group with the lowest antibody titers in agreement with Fioredda et al ${ }^{31}$. Therefore, revaccination with a booster dose should be indicated in patients with leukemia, especially after chemotherapy $^{32}$.

Unexpectedly, patients with previous IPD did not present greater anti-PCP IgG levels than patients without past IPD. A plausible explanation for this finding is that naturally acquired immunity depends on multiple factors as nasopharyngeal colonization or pneumococcal capsular antigens and surface proteins, whereas naturally acquired protection against IPD depends largely on antibodies against surface protein antigens rather than anti-capsular ones ${ }^{21}$. 
In our cohort, all the patients presented a high risk of developing pneumococcal disease and therefore, pneumococcal vaccination was indicated. However, our data showed that $70.6 \%$ of the patients lacked some dose, especially of polysaccharide vaccine, which agrees with similar series ${ }^{22,23}$. Thus, further efforts should be implemented to increase awareness and improve the rates of pneumococcal vaccination coverage in these patients.

Vaccine response is the product of correct vaccination coverage and of an adequate production of protective antibody titters. Therefore, even if the patient receives the recommended vaccine doses, an adequate immune system has to generate an antibody level high enough to prevent future pneumococcal infections. In our study, 12 of the 25 properly vaccinated patients did not reach an acceptable antibody level, as it has been found in other series ${ }^{24,25}$. This points out the low effectiveness of vaccination in children with underlying conditions affecting the immune system. In fact, we observed that responders presented higher levels of immunoglobulins, complement proteins and CD4/CD8 ratio than non-responders. These differences would explain why these patients produced higher pneumococcal titers after vaccination ${ }^{26}$.

IgG4 deficiency is the most common subclass of immunodeficiency, and $15 \%$ of normal children may present it. In our paper, we found that correct immunization was achieved by children with higher levels of IgG4. Our findings could explain Tian et al findings that link recurrent respiratory infections -particularly pulmonaryand bronchiectasis with IgG4 serum deficiency ${ }^{27}$.

In agreement with Avelino-Silva et al and Fuster et al, CD4/CD8 ratio was one of the most important predictors of vaccine response, and a lower ratio might be associated with impaired B cell function ${ }^{28,29}$. However, multiple elements such as environmental, behavioral, nutritional, intrinsic and extrinsic host factors influence vaccine response as well ${ }^{30}$.

Clinical and laboratory correlations between low CD4 T lymphocyte counts and C4 levels in patients with leukemia have been published ${ }^{33}$. The CD4 $\mathrm{T}$ lymphocyte is one of the main effectors of vaccine response, especially for conjugate vaccines. Thus, our LHT patients were prone to not responding to pneumococcal vaccines because their CD4 $\mathrm{T}$ lymphocyte counts were under $500 / \mathrm{mL}^{34}$. Interestingly, our LHT patients presented the higher $\mathrm{C} 4$ levels, although within normal range and should not be relevant ${ }^{35}$.

Finally, we were able to build a mathematical model to predict vaccination response with a $67 \%$ accuracy based on age, IT, C3, C4 IgG3 and lymphocyte levels. The model we suggest is simple enough to allow its use in clinical settings and to identify patients at higher risk of vaccination failure.

IT and lymphocyte counts are critical factors in the prediction of pneumococcal vaccine response ${ }^{36,37}$. In our research, IT was associated with lower response rates to pneumococcal vaccination while absolute lymphocyte count predicted a protective response ${ }^{38,39}$. The correlation between age and vaccination failure was also important, the mean age being higher in patients with primary vaccine failure. A possible explanation to this finding could be that younger patients were better vaccinated overall than older patients as a result of recent vaccination guidelines and risk awareness.

Although C3 plays a central role in the immune system, in our study high levels of this component were negatively associated with pneumococcal vaccine response. Recent studies suggest the close relationship between elevated levels of $\mathrm{C} 3$ and diabetes or obesity, diseases in which the role of the immune system has already been demonstrated ${ }^{40}$. On the other hand, elevated serum levels of C4 and IgG3 were correlated with vaccine response, which was consistent because both are essential components of the immune system ${ }^{36}$.

Limitations of the study include the relatively small number of patients recruited and the heterogeneity in the type of pneumococcal vaccines administered in the study population, which nonetheless, reflects the current situation of our patients.

In conclusion, we have studied pneumococcal vaccination coverage and vaccine response rates in children with underlying diseases. Our findings highlight the conditions and clinical data that should be taken into account to identify children at higher risk of vaccination failure. 


\section{ACKNOWLEDGEMENTS}

The authors express their sincere gratitude to all subjects included in the study.

\section{REFERENCES}

1. O'Brien KL, Wolfson LJ, Watt JP, et al. Burden of disease caused by Streptococcus pneumoniae in children younger than 5 years: global estimates. Lancet . 2009;374(9693):893-902.

2. World Health Organization.2020. Estimates of disease burden and costeffectiveness.http://www.who.int/immunization/monitoring_surveillance /burden/estimates/en/. Accessed 21 December 2020.

3. Backhaus E, Berg S, Andersson R, et al. Epidemiology of invasive pneumococcal infections: Manifestations, incidence and case fatality rate correlated to age, gender and risk factors. BMC Infect Dis . 2016;16(1):1-12.

4. Oligbu G, Collins S, Sheppard C, et al. Risk of Invasive Pneumococcal Disease in Children with Sickle Cell Disease in England: A National Observational Cohort Study, 2010-2015. Arch Dis Child . 2018;103(7):643-647.

5. Nuorti J. Whitney CG. Centers for Disease Control and Prevention (CDC). Prevention of pneumococcal disease among infants and children-use of 13 -valent pneumococcal conjugate vaccine and 23 -valent pneumococcal polysaccharide vaccine-recommendations of the Advisory Committee on Immunization Practices (ACIP) MMWR Recomm Rep. 2010;59:1-18.

6. Hsu KK, Shea KM, Stevenson AE, Pelton SI. Underlying conditions in children with invasive pneumococcal disease in the conjugate vaccine era. Pediatr Infect Dis J . 2011;30(3):251-253.

7. Comite Asesor de Vacunas (CAV-AEP). Neumococo. Manual de vacunas en linea de la AEP. http://vacunasaep.org/documentos/manual/cap-31. Accessed 10 November 2020.

8. Garrido-Jareno M, Puchades-Carrasco L, Orti-Perez L, A surface plasmon resonance based approach for measuring response to pneumococcal vaccine. Sci Rep . 2021;11(1):6502.

9. Balloch A, Licciardi PV, Tang MLK. Serotype-specific anti-pneumococcal IgG and immune competence: Critical differences in interpretation criteria when different methods are used. J Clin Immunol . 2013;33(2):335-341.

10. Oligbu G, Hsia Y, Folgori L, Collins S, Ladhani S. Pneumococcal conjugate vaccine failure in children: A systematic review of the literature. Vaccine . 2016;34(50):6126-6132.

11. Yucesoy B, Johnson VJ, Fluharty K, et al. Influence of cytokine gene variations on immunization to childhood vaccines. Vaccine . 2009;27(50):6991-6997.

12. Torres NV, Santos G. The (mathematical) modeling process in biosciences. Front Genet. 2015;22(6):19.

13. Motta S, Pappalardo F. Mathematical modeling of biological systems. Brief Bioinform . 2013;14(4):411422.

14. Le Polain De Waroux O, Edmunds WJ, Takahashi K, et al. Predicting the impact of pneumococcal conjugate vaccine Programme options in Vietnam.Hum Vaccines Immunother . 2018;14(8):1939-1947.

15. Choi YH, Andrews N, Miller E. Estimated impact of revising the 13-valent pneumococcal conjugate vaccine schedule from $2+1$ to 1+1 in England and Wales: A modelling study. PLoS Med . 2019;16(7):130.

16. Schuerman L, Wysocki J, Tejedor JC, Knuf M, Kim KH, Poolman J. Prediction of pneumococcal conjugate vaccine effectiveness against invasive pneumococcal disease using opsonophagocytic activity and antibody concentrations determined by enzyme-linked immunosorbent assay with $22 \mathrm{~F}$ adsorption. Clin Vaccine Immunol . 2011;18(12):2161-2167.

17. Schauer U, Stemberg F, Rieger CHL, et al. Levels of Antibodies Specific to Tetanus Toxoid, Haemophilus influenzae type b, and pneumococcal capsular polysaccharide in healthy children and adults. Clin Diagn Lab Immunol . 2003;10(2):202-207.

18. Sanchez-Ramon S, de Gracia J, Garcia-Alonso AM, et al. Multicenter study for the evaluation of the antibody response against salmonella typhi Vi vaccination (EMPATHY) for the diagnosis of Antipolysaccharide antibody production deficiency in patients with primary immunodeficiency. Clin Im- 
munol . 2016;169:80-84.

19. Alvarez Garcia FJ, Cilleruelo Ortega MJ, Alvarez Aldean J, et al. [Immunisation schedule of the Spanish Association of Paediatrics: 2020 recommendations]. An Pediatr (Barc) . 2020;92(1):52 e1e10.

20. Hernandez S, Moraga-Llop F, Diaz A, et al. Failures of 13-valent conjugated pneumococcal vaccine in age-appropriately vaccinated children 2-59 months of age, Spain. Emerg Infect Dis . 2020;26(6):11471155.

21. Wilson R, Cohen JM, Reglinski M, et al. Naturally Acquired Human Immunity to Pneumococcus Is Dependent on Antibody to Protein Antigens.PLoS Pathog . 2017;13(1):1-26.

22. Schmedt N, Schiffner-Rohe J, Sprenger R, Walker J, Von Eiff C, Hackl D. Pneumococcal vaccination rates in immunocompromised patients-A cohort study based on claims data from more than 200,000 patients in Germany. PLoS One . 2019;14(8):1-15.

23. Reeves SL, Jary HK, Gondhi JP, Kleyn M, Wagner AL, Dombkowski KJ. Pneumococcal vaccination coverage among children with sickle cell anemia, sickle cell trait, and normal hemoglobin. Pediatr Blood Cancer . 2018;65(10):e27282.

24. Nero AC, Akuete K, Reeves SL, Dombkowski KJ. Pneumococcal vaccination rates in children with sickle cell disease. J Public Heal Manag Pract . 2014;20(6):587-590.

25. Stray-Pedersen A, Aaberge IS, Fruh A, Abrahamsen TG. Pneumococcal conjugate vaccine followed by pneumococcal polysaccharide vaccine; immunogenicity in patients with ataxia-telangiectasia. Clin Exp Immunol . 2005;140(3):507-516.

26. Chinn IK, Orange JS. Immunodeficiency Disorders. Pediatr Rev . 2019;40(5):229-242.

27. Tian X, Deng Z, Wang S, Wang Y. Basic Research and Clinical Reports Associated with Low Serum IgG4 Concentrations. Int Arch Allergy Immunol . 2020;181(2):149-158.

28. Avelino-Silva VI, Miyaji KT, Hunt PW, et al. CD4/CD8 Ratio and KT Ratio Predict Yellow Fever Vaccine Immunogenicity in HIV-Infected Patients. PLoS Negl Trop Dis. 2016;10(12):1-15.

29. Fuster F, Vargas JI, Jensen D, et al. CD4/CD8 ratio as a predictor of the response to HBV vaccination in HIV-positive patients: A prospective cohort study. Vaccine. 2016;34(16):1889-1895.

30. Zimmermann P, Curtis N. Factors That Influence the Immune Response to Vaccination. Clin Microbiol Rev . 2019;32(2):e00084-18.

31. Fioredda F. Immunity against hepatitis B and measles vaccination after chemotherapy for acute lymphoblastic leukaemia in children: Revaccination policy. Rev Bras Hematol Hemoter . 2012;34(4):258259.

32. Dorval S, Coic L, Blais D, et al. Pneumococcal Vaccination During Chemotherapy in Children Treated for Acute Lymphoblastic Leukemia. Open Forum Infect Dis. 2019;6(2):S949.

33. Middleton O, Cosimo E, Dobbin E, et al. Complement deficiencies limit CD20 monoclonal antibody treatment efficacy in CLL. Leukemia.2015;29(1):107-114.

34. Gonzalez-Rodriguez AP, Contesti J, Huergo-Zapico L, et al. Prognostic significance of CD8 and CD4 T cells in chronic lymphocytic leukemia.Leuk Lymphoma. 2010;51(10):1829-1836.

35. Garcia-Prat M, Vila-Pijoan G, Martos Gutierrez S, et al. Age-specific pediatric references ranges for immunoglobulins and complement proteins on the Optilite TMautomated turbidimetric analyzer. $J$ Clin Lab Anal . 2018;32(6):e22420.

36. Pulendran B. Systems vaccinology: probing humanity's diverse immune systems with vaccines. Proc Natl Acad Sci USA . 2014;111(34):12300-12306.

37. Mavinkurve-Groothuis AMC, Van Der Flier M, Stelma F, Van Leer-Buter C, Preijers FW, Hoogerbrugge PM. Absolute lymphocyte count predicts the response to new influenza virus H1N1 vaccination in pediatric cancer patients. Clin Vaccine Immunol . 2013;20(1):118-121.

38. Cao Y, Zhao D, Xu AT, Shen J, Ran ZH. Effects of immunosuppressants on immune response to vaccine in inflammatory bowel disease. Chin Med J (Engl). 2015;128(6):835-838.

39. Aringer M. Vaccination under TNF blockade-less effective, but worthwhile. Arthritis Res Ther. 2012;14(3):117.

40. Borne Y, Muhammad IF, Lores-Motta L, et al. Complement C3 associates with incidence of diabetes, 
but no evidence of a causal relationship.J Clin Endocrinol Metab. 2017;102(12):4477-4485.

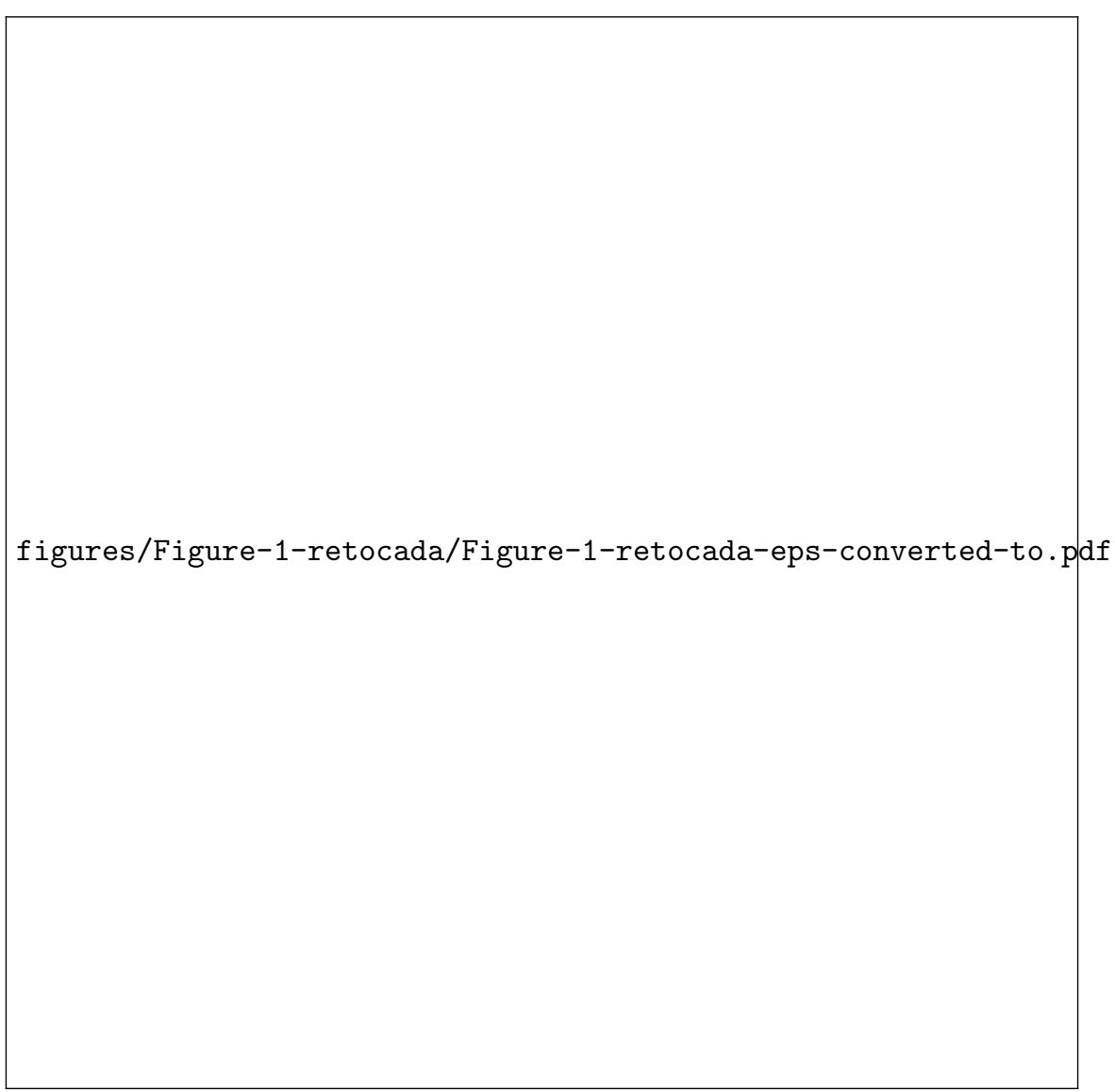


figures/FIGURA-2-RETOCADA/FIGURA-2-RETOCADA-eps-converted-to.pdf

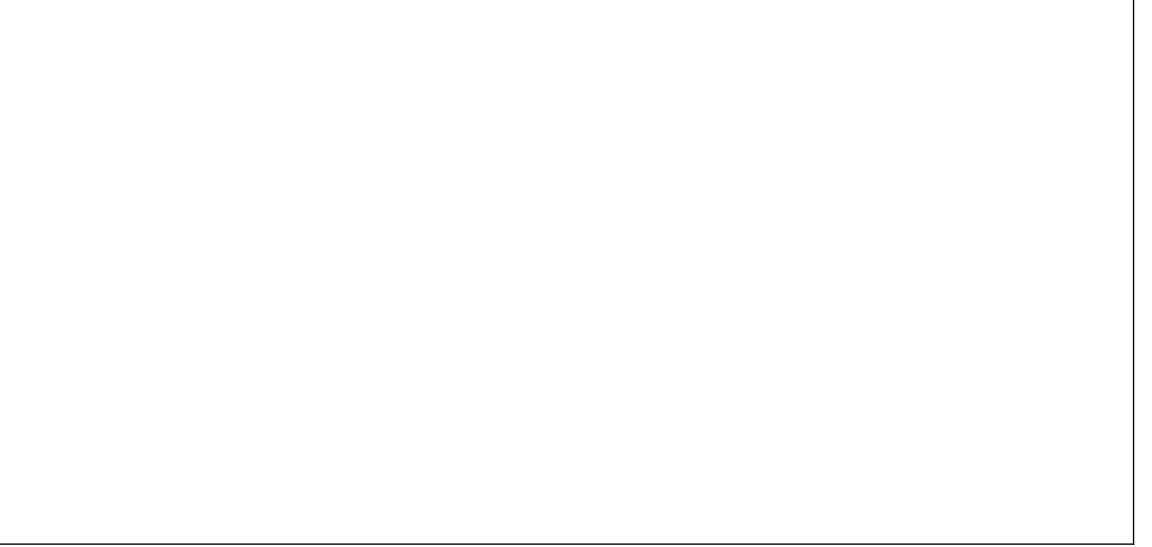

Hosted file

Tablas.pdf available at https://authorea.com/users/413025/articles/521488-study-ofpneumococcal-vaccine-response-in-children-with-underlying-medical-conditions 